\title{
Suture clutter can be prevented by a simple method in delayed anastomosis during the Turnbull-Cutait operation
}

\author{
E. Gedik • A. M. Kuzu
}

Received: 2 February 2009/Accepted: 7 July 2009/Published online: 4 February 2010

(C) Springer-Verlag 2010

The Turnbull-Cutait abdominoperineal pull-through procedure with delayed coloanal anastomosis is used as a salvage procedure in patients with complex anorectal problems that might otherwise require permanent stoma. This procedure is a vialable option in patients with a reoperated, irradiated pelvis with chronic inflammation or infection due to anastomotic leakage, rectovaginal or rectourethral fistula, technical difficulties related to complicated rectal cancer, or other septic conditions.

A simple method to prevent suture clutter in a 57-yearold woman, who underwent an abdominoperineal pullthrough procedure with delayed coloanal anastomosis, is presented. She had had anastomotic leakage and chronic pelvic sepsis following low anterior resection with protective ileostomy after neo-adjuvant radiochemotherapy for rectal carcinoma 14 months prior to surgery. Suture clutter can easily lead to prolongation of the operation and to confusion in the delayed Turnbull-Cutait anastomosis. We describe a novel method to prevent suture clutter (Figs. 1-9).

The patient had no postoperative complications and was discharged on postoperative day 12. Bladder and sexual functions were normal and the Wexner continence score was 4.0 six months after discharge.

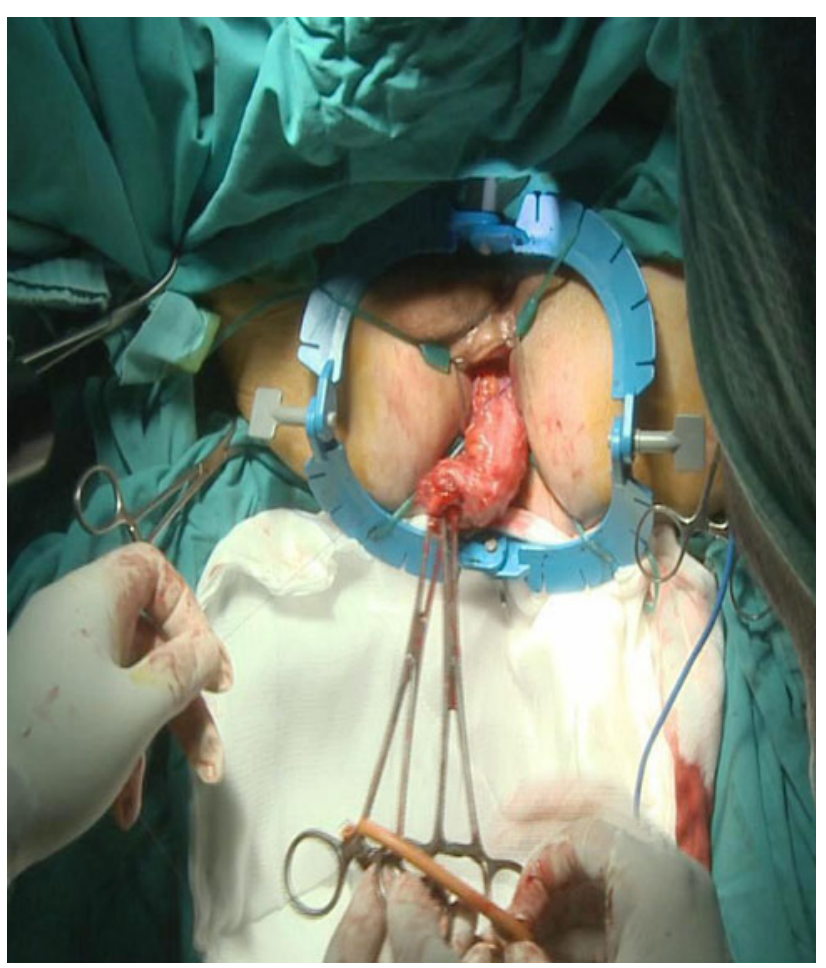

Fig. 1 Pull-through of the descending colon 


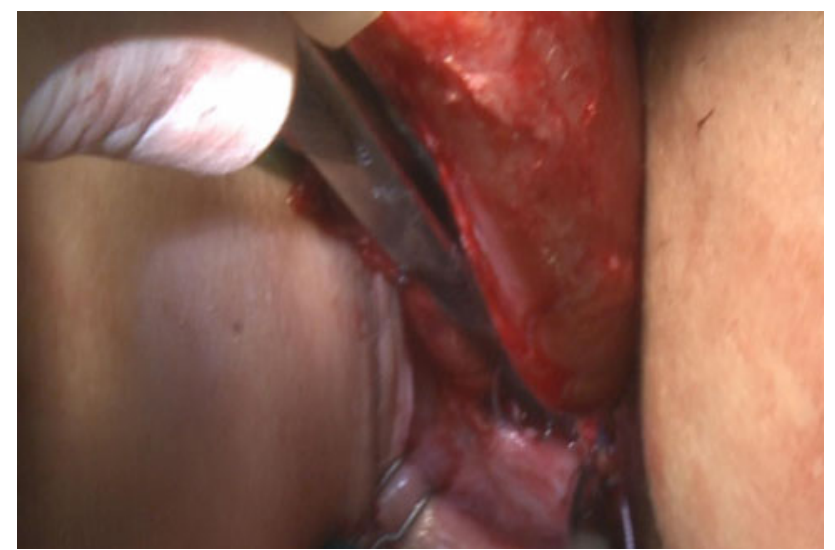

Fig. 2 The recoiling of the colon is prevented by intersphincteric stay sutures

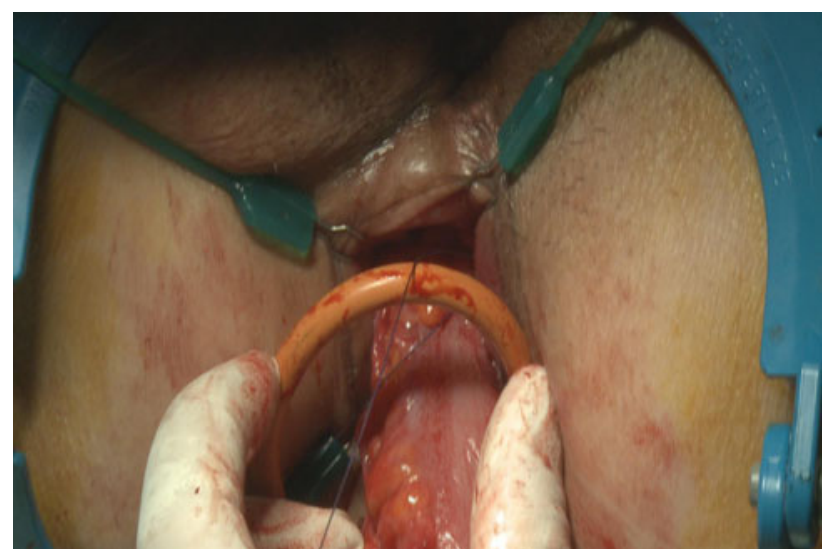

Fig. 3 A rounded silicon drain is wrapped around the colon. Sutures are passed through the mucosa and the silicon drain

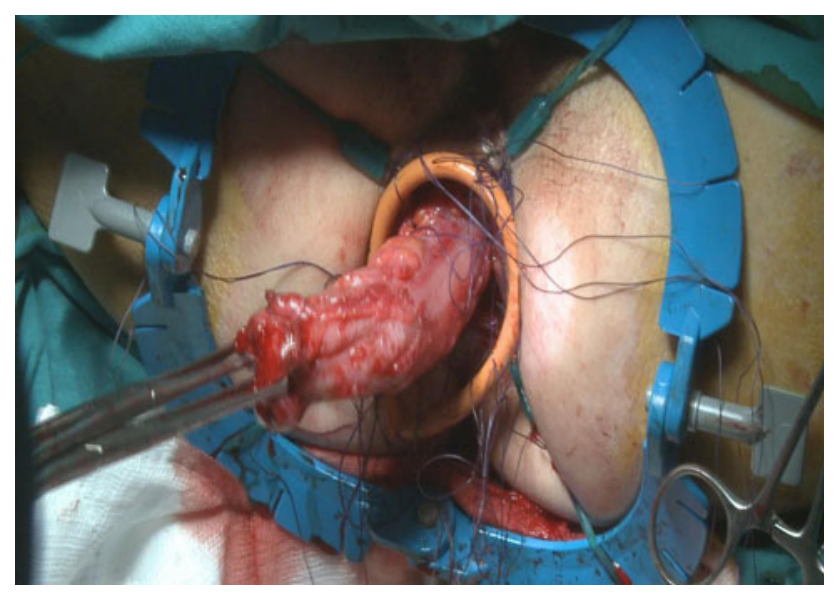

Fig. 4 Suture placement is completed sequentially in a clockwise direction

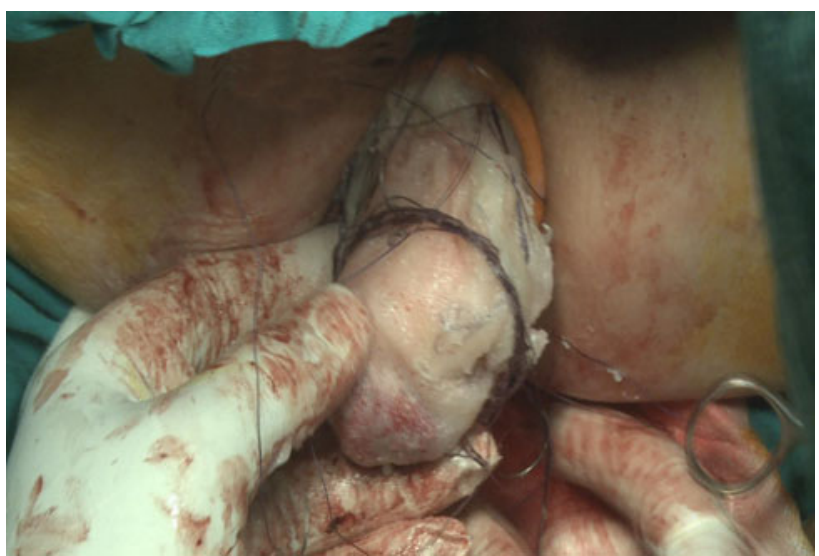

Fig. 5 The colon is wrapped with a vaseline pad, and suture materials are also wrapped around the vaseline pad in the same direction

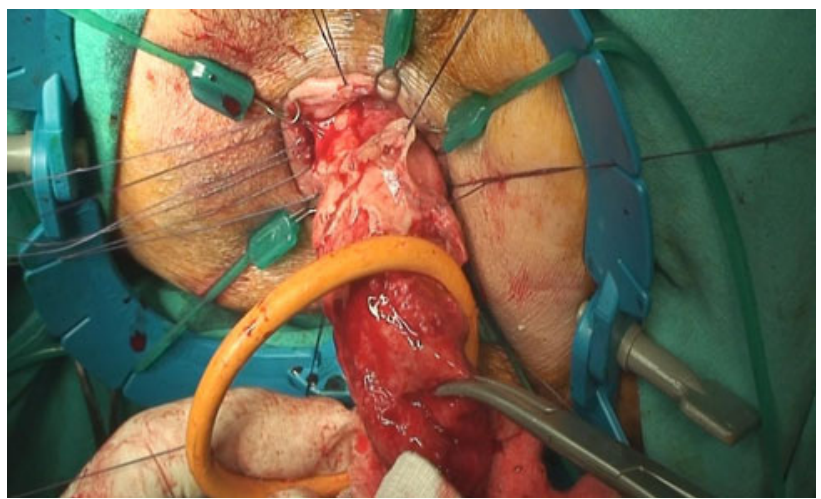

Fig. 6 Sutures in the silicon drain are unstitched one by one in the second stage of the operation within 5 to 7 days

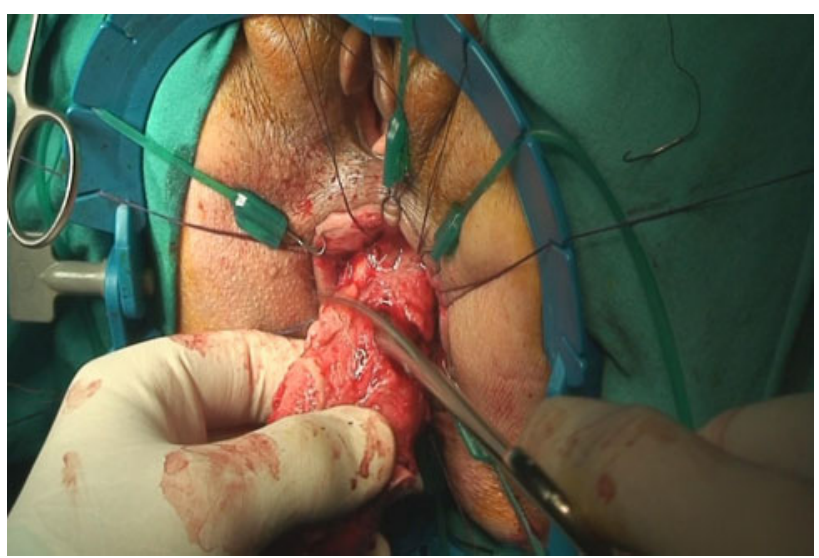

Fig. 7 The silicon drain is taken out and the redundant colon is resected 


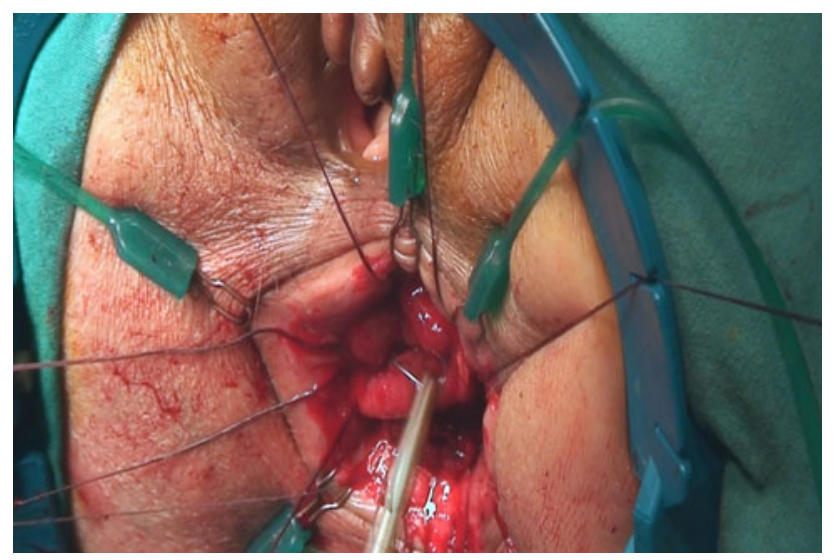

Fig. 8 The sutures are used to fashion a coloanal anastomosis

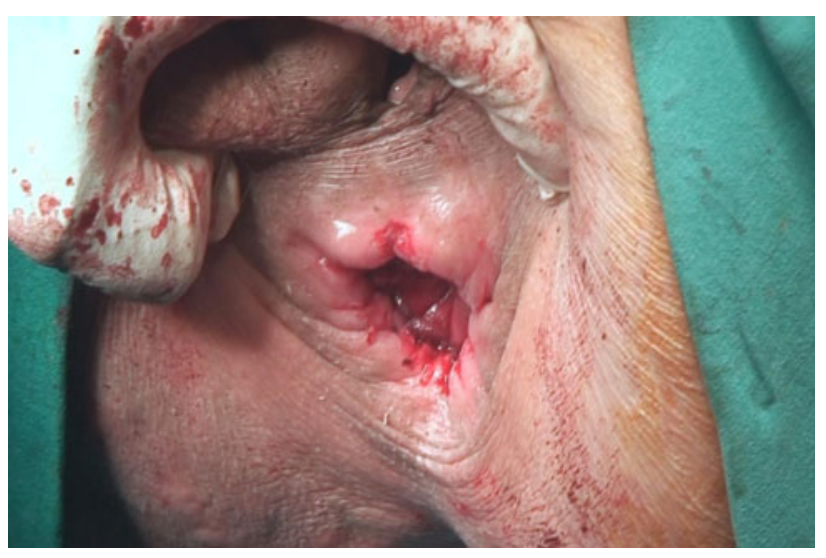

Fig. 9 Coloanal anastomosis is completed 